\title{
Immunohistochemical Study of p53 Expression in Colorectal Adenocarcinomas and its Clinicopathological Correlation
}

\author{
Varshaa Chithrra*, Jaison Jacob John, Shivashekar Ganapathy and Veena Raja
}

Department.of Pathology, SRM Medical College Hospital and Research Centre, Kattankulathur, Chennai-603203, TamilNadu, India

\section{ABSTRACT}

Background: Colorectal carcinoma is the fourth most frequent cancer in men and the third in women worldwide. The identification of molecular markers that have a prognostic significance in colorectal carcinoma may have an impact on its treatment. This study aims to identify and evaluate the immunohistochemical expression of p53 in colorectal adenocarcinomas and to correlate its expression with various clinicopathological parameters.

Methods: The present study was an observational study done in 30 surgical resection specimens received in the Department of Pathology, SRM Medical College and Research Centre, Kattankulathur, Tamil Nadu from June 2015-June 2017. Clinical history and clinicopathological parameters were obtained ,histomorphological and immunohistochemical evaluation was done and the results were correlated with clinicopathological variables. The IHC panel we used was mouse monoclonal p53 antibody (PathnSitu).

Result: In our study, p53 overexpression was noted in 70\% cases of colorectal adenocarcinomas. p53 overexpression was noted more in conventional adenocarcinomas $(85.7 \%)$, more in left sided colonic carcinomas $(71.4 \%)$, in advanced pT stage (57.1\% in pT3) and also in cases with lymph node metastasis $(57.1 \%)$. No statistically significant correlation of p53 with other clinicopathological variables such as age, gender, nature of specimen, tumor site, size, histological type, grade, tumor perforation, bowel wall invasion and perineural invasion were noted.

Conclusion: Our study supports that p53 overexpression is an indicator of poor prognosis and may prove as an useful marker in identifying high risk patients who may benefit from adjuvant therapy in the early stage of disease.

\section{Keywords: Colorectal Adenocarcinomas, p53, Immunohistochemistry}

\section{Introduction}

Colorectal carcinoma (CRC) is the fourth most frequent cancer in men $(746,000$ cases, $10.0 \%$ of the total) and the third in women $(614,000$ cases, $9.2 \%$ of the total) worldwide. ${ }^{[1]}$ The global burden of colorectal carcinoma is expected to increase by $60 \%$ to more than 2.2 million new cases and 1.1 million deaths by $2030 .{ }^{[2]} \mathrm{CRC}$ is a relatively uncommon malignancy in India when compared with the western world. However the changing demographic and epidemiological patterns noted over the past few years in the Indian population may contribute in future to an increased CRC risk in this country. The ASR of CRC in India have been estimated to be 4.2 and 3.2/100,000 for males and females. ${ }^{[3]}$ The ASR of Colorectal Cancer in Chennai have been estimated to be 1.9 for colon and 2.6 for rectal cancers in males and in females it is 1.8 for colon and 1.9 for rectal cancers. ${ }^{[4]}$

The identification of molecular markers that have a prognostic significance in colorectal carcinoma may have an impact on treatment and surveillance. The objective of our study was to evaluate the immunohistochemical expression of $\mathrm{p} 53$ in colorectal adenocarcinomas and to correlate its expression with various clinicopathological parameters.

\section{Materials and Methods}

This observational study was done on 30 specimens of colorectal adenocarcinomas received in Department of Pathology, SRM Medical college and Research centre, Kattankulathur,Tamil Nadu from June 2015 to June 2017. Institutional Ethical Committee granted approval for the study. Inclusion criteria was all surgical resection specimens of right and left Hemicolectomy (RHC , LHC), Sigmoid Colectomy (SC), Low Anterior Resection (LAR), Abdominoperineal resection (APR) and Total colectomy specimens (TC) .Exclusion criteria included Colonoscopic biopsies, cases with extensive tumor necrosis and no viable tumor cells for IHC study.The detailed clinical history and clinicopathological parameters like age, gender, clinical presentation, tumor site, tumor size and gross morphology were collected or abstracted in all cases from the patients case files and from pathology reports. For prospective cases all the resection specimens were received in Pathology Department in 10\% Formalin. In every case the standard protocol for surgical grossing of 
resected specimens was followed. For further analysis the anatomic locations were grouped into right colon (from caecum to transverse colon) and left colon (from splenic flexure to sigmoid colon and rectum). For retrospective cases the histopathology reports, paraffin blocks and slides best representing the tumor in each patient were retrieved from the archives. After conventional processing, from each paraffin block of tumor tissue, three sections were taken. One section of $5 \mathrm{Um}$ thickness were stained by Haematoxylin and Eosin $(\mathrm{H} \& \mathrm{E})$ for histopathological study and other 2 sections of $4 \mathrm{Um}$ thickness were taken on a glass slide coated with adhesive Amino Propyl Triethoxy Silane (APES) for IHC to detect p53 expression. Sections were also cut from the retrieved paraffin blocks in this similar manner.The H\&E stained slides were studied by light microscopy for histological diagnosis and assessment of differentiation, lymphovascular, perineural invasion, lymph node metastasis and TNM staging. IHC is performed by Indirect method with microwave antigen retrieval on 2 sections. Positive controls were run with each batch of slides. A colorectal neoplasm with score $3+$ p53 overexpression was used as positive control for p53 in all slide runs. In our study we considered only tumor cells with distinct nuclear immunostaining for p53 in atleast $10 \%$ of all malignant cells in a tissue section as positive. We chose the cut off value of $10 \%$ positivity as this value showed the highest concordance between IHC detection of nuclear accumulation $\mathrm{p} 53$ and point mutations of p53 gene detected by Single stranded conformation polymorphism analysis. ${ }^{[5]}$ p53 immunostaining were scored in a semiquantitative manner, ${ }^{\left[{ }^{[6]}\right.}$ as follows : $0-<10 \%, 1+$ $10-25 \%, 2+-25-50 \%, 3+->50 \%$ positive cells. Primary data entered in MS Excel and analyzed using computer software, Statistical Package for Social Sciences (SPSS) version 20. To elucidate the associations and comparisons between different parameters, Chi square test was used at $5 \%$ level of significance. For all statistical evaluations a $\mathrm{p}$ value $<0.05$ was considered significant.

\section{Results:}

Clinicopathological Data: Age of the patients in this study ranged from 30-88 years. Majority were in age of $50-59$ years $(40 \%)$. Majority were males $18(60 \%)$ and females were $12(40 \%)$. The male to female ratio was 1.5 : 1. Commonest symptom was Bleeding per rectum $(60 \%)$ followed by constipation (40\%), abdominal pain and weight loss (36.7\%), fever of unknown origin (13.3\%) and hematochezia (10\%). Among the 30 specimens studied, 12 (40\%) were RHC , 7 (23.3\%) were APR , $6(20 \%)$ were LHC, 4 (13.3\%) were LAR and 1 (3.3\%) was SC. Rectum $(33.3 \%)$ was the commonest site affected by the tumor followed by Caecum (16.7\%) and Sigmoid colon (16.7\%), Ascending colon(13.3\%),hepatic flexure (6.7\%),
Rectosigmoid junction (6.7\%) ,Caecum and ileocaecal junction $(3.3 \%)$ and Splenic flexure (3.3\%). Majority of the tumors were located in the left side of the colon -18 $(60 \%)$ and $12(40 \%)$ were located in the right side of the colon. 12 cases $(40 \%)$ had tumor size $>5 \mathrm{~cm}$ and 11 cases (36.7\%) had tumor size $<5 \mathrm{~cm}$ and 7 cases $(23.3 \%)$ had a tumor size of $5 \mathrm{~cm} .25$ cases $(83.3 \%)$ were adenocarcinoma -conventional type and 5 cases $(16.7 \%)$ were mucinous type. 15 cases $(50 \%)$ were moderately differentiated (MD) /Grade II tumors , 12 cases (40\%) were well differentiated (WD)/Grade I tumors and 3 cases (10\%) were poorly differentiated (PD)/Grade III tumors. Macroscopic tumor perforation were present in 2 cases $(6.7 \%)$ and were absent in 28 cases $(93.3 \%)$. Lymphnode metastasis were noted in 14 cases $(46.7 \%)$ and were absent in 16 cases $(53.3 \%) .19$ cases $(63.3 \%)$ exhibited T3 stage and 11 cases(36.7\%) exhibited T2 stage. 2 cases $(6.6 \%)$ exhibited NX stage, 14 cases $(46.7 \%)$ exhibited N0 stage, 9 cases (30\%) in N1 stage, 5 cases $(16.7 \%)$ each exhibited N2 stage. Lymphovascular invasion was identified in 4 cases(13.3\%) and was absent in 26 cases $(86.7 \%)$. Perineural invasion were noted in 3 cases $(10 \%)$ and were absent in 27 cases $(90 \%)$.

IHC Results with Clinicopathological Correlation: p53 IHC staining were positive in 21 cases $(70 \%)$ and were negative in 9 cases (30\%). p53 IHC scoring(Table 1) .p53 positivity in males were $61.9 \%$ and in females it was $38.1 \%$. p53 positivity were noted in $71.4 \%$ of left sided colonic tumors and $28.6 \%$ in right sided colonic tumors ( Table 2). Out of the 25 conventional adenocarcinoma cases $18(85.7 \%)$ were positive for p53 and $7(77.8 \%)$ were p53 negative. Of the 5 mucinous adenocarcinoma cases $3(14.3 \%)$ were positive for p53 and $2(22.2 \%)$ were negative for $\mathrm{p} 53$. Among the $12 \mathrm{WD}$ tumors -9 cases $(42.9 \%)$ were p53 positive and 3 cases $(33.3 \%)$ were p53 negative. Out of the 15 MD tumors- 11 cases (52.4 $\%)$ were p53 positive and 4 cases (44.4\%) were p53 negative. Among the 3 PD tumors -1 case $(4.8 \%)$ was p53 positive and 2 cases $(22.2 \%)$ were p53 negative. Out of the 2 cases which had macroscopic tumor perforation, p53 were positive in 2 cases (9.5\%). Rest of the 28 cases which had no macroscopic tumor perforation, p53 was positive in 19 cases $(90.5 \%)$ and negative in 9 cases (28 $\%)$. Out of the 14 cases which had lymph node metastasis, 12 cases $(57.1 \%)$ were p53 positive and 2 cases $(22.2 \%)$ were p53 negative (Table 3). Among the 16 cases which had no lymph node metastasis, 9 cases $(42.9 \%)$ were p53 positive and 7 cases $(77.8 \%$ ) were p53 negative. Out of the 11 cases with T2 level of tumor invasion, 9 cases (42.9\%) were $\mathrm{p} 53$ positive and 2 cases $(22.2 \%)$ were $\mathrm{p} 53$ negative. Out of the 19 cases with T3 level of invasion, p53 was positive in 12 cases $(57.1 \%)$ and negative in 7 cases $(77.8$ $\%$ ).p53 and lymph node status (Table 2). Of the 4 cases 
which showed lymphovascular invasion, 1 case $(4.8 \%)$ was p53 positive and 3 cases(33.3\%) were p53 negative. Rest of the 26 cases in which no lymphovascular invasion was identified , 20 cases $(95.2 \%)$ were p53 positive and 6 cases $(66.7 \%)$ were $\mathrm{p} 53$ negative. All these findings were statistically significant with a $p$ value of 0.03 .
Out of the 3 cases in which perineural invasion was identified, 1 case $(4.8 \%)$ was positive for $\mathrm{p} 53$ and 2 cases $(22.2 \%)$ were negative for p53.Among the 27 cases in which perineural invasion was not identified, 20 cases $(95.2 \%)$ were p53 positive and 7 cases $(77.8 \%)$ were p53 negative.

Table 1: Frequency of p53 expression in colorectal adenocarcinoma cases.

\begin{tabular}{|c|c|c|}
\hline p53 SCORING & Frequency & Percent \\
\hline 0 & 9 & 30 \\
\hline $1+$ & 4 & 13.3 \\
\hline $2+$ & 9 & 30 \\
\hline $3+$ & 8 & 26.7 \\
\hline Total & 30 & 100 \\
\hline
\end{tabular}

Table 2: p53 and tumor with respect to the side of colon affected

\begin{tabular}{|c|c|c|c|c|c|c|c|}
\hline \multirow{2}{*}{ TUMOR SIDE } & \multicolumn{5}{|c|}{ p53 } & \multirow{2}{*}{ Chi sq } & \multirow{2}{*}{$p$} \\
\hline & POSITIVE & $\%$ & NEGATIVE & $\%$ & Total & & \\
\hline LEFT SIDED & 15 & 71.4 & 3 & 33.3 & 18 & \multirow{3}{*}{3.81} & \multirow{3}{*}{0.05} \\
\hline RIGHT SIDED & 6 & 28.6 & 6 & 66.7 & 12 & & \\
\hline Total & 21 & 100.0 & 9 & 100.0 & 30 & & \\
\hline
\end{tabular}

Table 3: p53 and lymph node metastasis.

\begin{tabular}{|c|c|c|c|c|c|c|c|}
\hline \multirow{2}{*}{$\begin{array}{l}\text { LYMPH NODE } \\
\text { METASTASIS }\end{array}$} & \multicolumn{5}{|c|}{ p53 } & \multirow{2}{*}{ Chi sq } & \multirow{2}{*}{$p$} \\
\hline & POSITIVE & $\%$ & NEGATIVE & $\%$ & Total & & \\
\hline PRESENT & 12 & 57.1 & 2 & 22.2 & 14 & \multirow{3}{*}{3.09} & \multirow{3}{*}{0.07} \\
\hline ABSENT & 9 & 42.9 & 7 & 77.8 & 16 & & \\
\hline Total & 21 & 100.0 & 9 & 100.0 & 30 & & \\
\hline
\end{tabular}

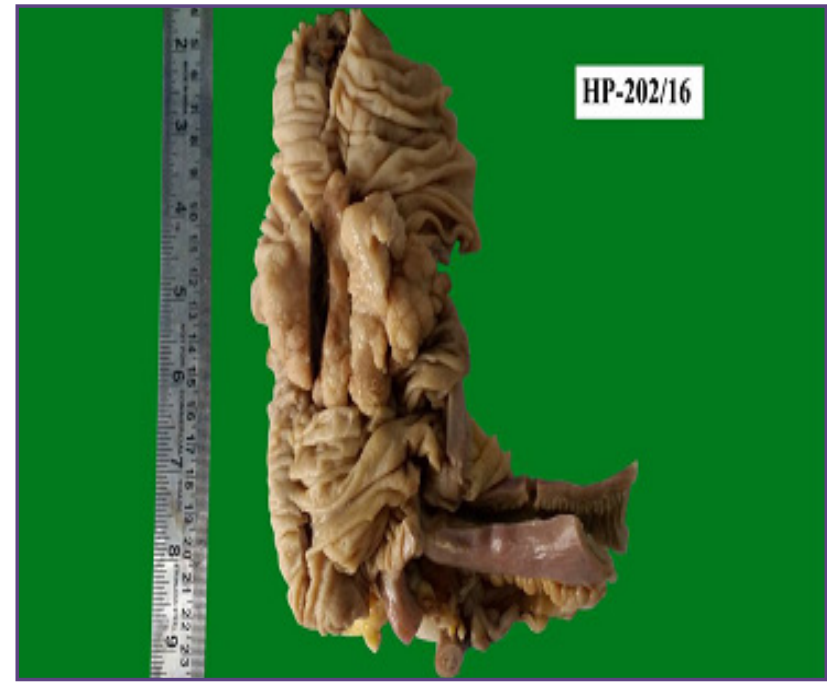

Fig. 1: Gross picture of Right Hemicolectomy showing tumor in the Caecum.

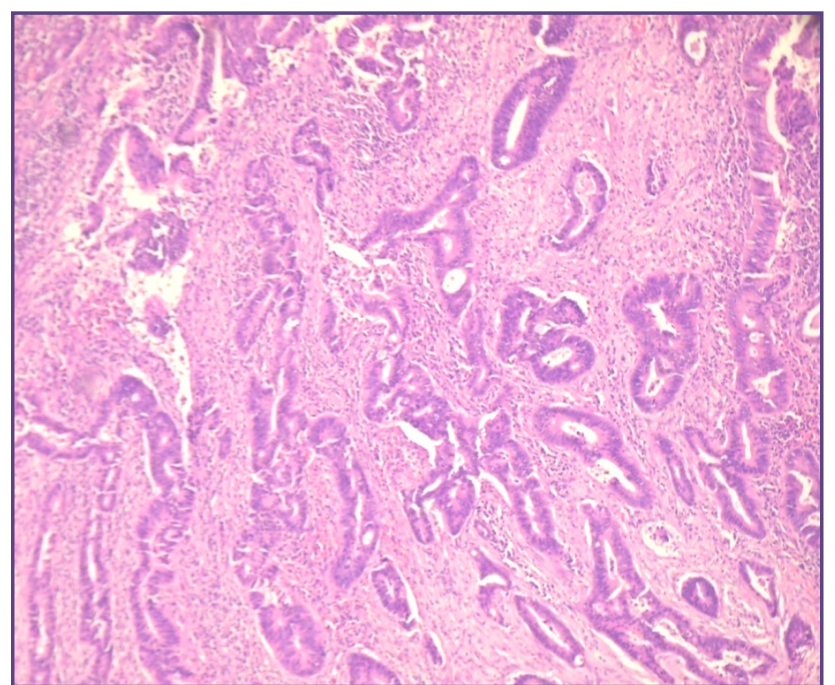

Fig. 2: Adenocarcinoma Rectum -Moderately differentiated (H\&E,x 100). 


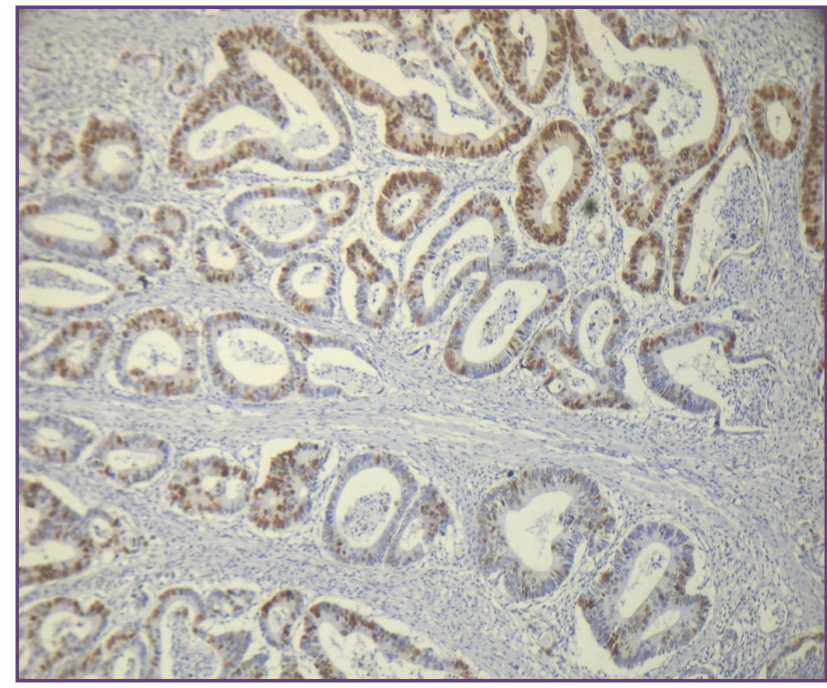

Fig. 3: p53 overexpression in tumor cells;Score 1+ (IHC $\mathrm{x} 100)$.

\section{Discussion}

Although many studies have clarified the role of molecular markers in colorectal malignancy, there is a little data in our geographical region, Kattankulathur, Tamilnadu. So we attempted to study the immunohistochemical expression of p53 in colorectal adenocarcinomas in this area and tried to correlate the expression pattern of these markers with various clinicopathological parameters.

In our study mean age of the patients was $56.63+/-14.03$ which was in concordance with the study by Kavitha Mardi ${ }^{[7]}$ and Hunaldo Lima de Menzes. ${ }^{[8]}$ Male to female ratio was $1.5: 1$ which were in concordance with the studies by Chandrakumar Shanmugham , ${ }^{[10]}$ Guru Raja Prasad ${ }^{[11]}$ and Claudia Valentina Georgescu. ${ }^{[9]}$ Majority of the tumors were located in the left side of the colon $-18(60 \%)$ and 12 $(40 \%)$ were located in the right side of the colon, which were in concordance with the studies by Azza Hegazy , ${ }^{[12]}$ Kavitha Mardi , ${ }^{[7]}$ Guru Raja Prasad. [11] Usually tumor size is recorded for the purpose of documentation ,however tumor size is not related to outcome. A few studies have shown that tumor size is of no prognostic significance in colorectal cancer. ${ }^{[13,14]} 25$ cases $(83.3 \%)$ were adenocarcinoma -conventional type and 5 cases $(16.7 \%)$ were mucinous type. Study by Kavitha Mardi, ${ }^{[7]}$ showed similar distribution of histological type . We also found that conventional adenocarcinomas were noted more in the left colon and mucinous adenocarcinomas were noted more in the right colon, which is an established fact as per the literature. Majority of the cases in our study were moderately differentiated (50\%) which was in concordance with the study done by Frank A Sinicrope. ${ }^{[15]}$

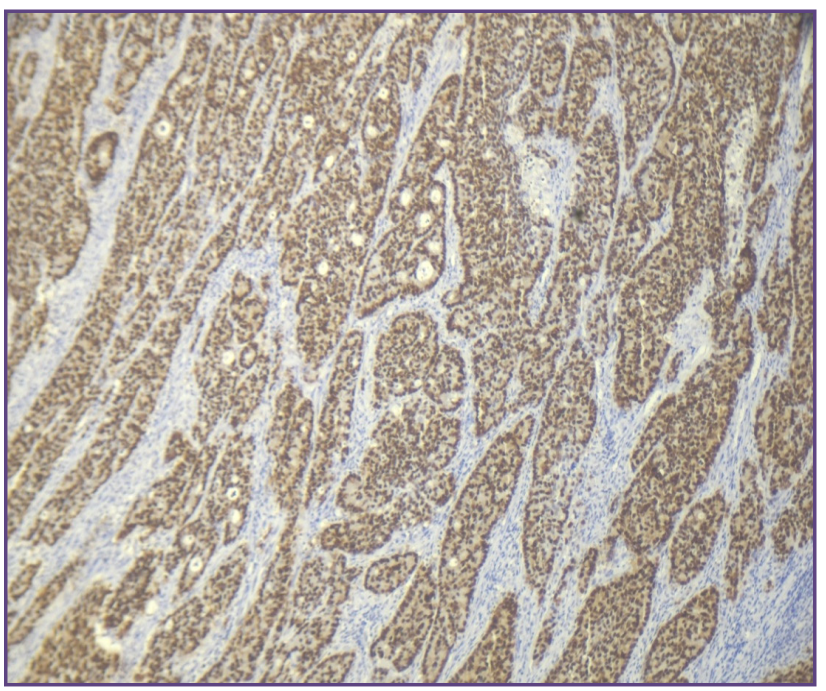

Fig. 4 : p53 overexpression in tumor cells;Score 3+ (IHC $\mathrm{x} 100$ ).

Tumor perforation is an uncommon complication of colorectal cancer and is associated with poor outcome. In our study 2 cases $(6.7 \%)$ showed macroscopic tumor perforation and 28 cases( $93.3 \%)$ were intact. The 2 cases which showed tumor perforation were noted in elderly patients with 65 years and 76 years of age. Various studies have reported tumor perforation rates ranging from $2.6 \%$ to $9 \%$ and also were noted to occur in older patients which was in concordance with our study. ${ }^{[16]}$

Lymph node assessment remains mainstay of deciding prognosis and adjuvant chemotherapy. We noted lymph node metastasis in 14 cases (46.7\%) and were absent in 16 cases (53.3\%) which were in concordance with studies by Azza Hegazy ${ }^{[12]}$ and Guru Raja Prasad. ${ }^{[11]} 19$ cases $(63.3 \%)$ exhibited pT3 stage and 11 cases (36.7\%) exhibited pT2 stage which were in concordance with study by Guru Raja Prasad. ${ }^{[1]}$

Various studies have reported that lymphovascular invasion were significantly associated with the degree of lymph node metastasis and also is a key factor in predicting the prognosis. ${ }^{[17,18]}$ In our study lymphovascular invasion was identified in 4 cases (13.3\%) and only 1 case with lymphovascular invasion showed lymph node metastasis. So Lymphovascular invasion is useful in identifying highrisk patients with node-negative tumors warranting the need for an adjuvant chemotherapy.

Study by Pages. $F^{[19]}$ showed that Perineural invasion was consistently associated with poor differentiation, advanced pT stage, lymphovascular invasion, which in turn determines the aggressive behaviour in CRC. We noted 
perineural invasion in 3 cases $(10 \%)$ and all the 3 cases showed pT3 Stage and lymphovascular invasion which was in concordance with Pages. F study. ${ }^{[19]}$ However only 1 case showed poor differentiation of the tumor.

Studies by Mulder [20] and Belluco et al [21] showed overexpression of p53 nuclear protein varies from 40-72 $\%$ in CRC and is inconsistent. This discrepancy could be due to interstudy variations including choice of the IHC methods, sensitivity and specificity of different antibodies used, different scoring systems and cut off values used for interpretations of the results and difference in study population . In our study, p53 overexpression were noted in 21 cases $(70 \%)$ and were negative in 9 cases $(30 \%)$,which was in concordance with studies by Simonetta Buglioni et al ${ }^{[22]}$ and Komal Mahendra Ashar. ${ }^{[23]}$

Out of the 21 p53 positive cases, p53 overexpression were noted in 18 cases $(85.7 \%)$ of conventional adenocarcinomas and in 3 cases $(14.3 \%)$ of the 5 mucinous adenocarcinomas .This was in concordance with studies conducted by Caludia Valentina Georgescu ${ }^{[9]}$ and Giovanni Lanza J R et al . ${ }^{[24]}$ However this result failed to reach the level of stastistical significance. We also noted that conventional adenocarcinomas were noted more in the left colon and mucinous adenocarcinomas were noted in the right colon which is an established fact as per the literature . Our findings on p53 overexpression in conventional adenocarcinomas support the suggestion that mucinous carcinomas may arise through a different pathogenetic mechanisms.

In our study nuclear p53 overexpression were detected in $71.4 \%$ of left sided colonic carcinomas and $28.6 \%$ of right sided colonic carcinomas. This was in concordance with Ofner. D et al study, ${ }^{[25]}$ Frank A.Sinicrope study ${ }^{[15]}$ and Guru Raja Prasad study. ${ }^{[11]}$ However there was no statistical significance. Our findings of p53 overexpression with respect to the side of the colon suggests that left sided colonic carcinomas and right sided colonic carcinomas may differ in their genetic susceptibities to neoplastic transformation .

We noted p53 overexpression in advanced pT stage, $57.1 \%$ in pT3 stage as opposed to $42.9 \%$ in pT2 stage. Similar p53 overexpression were also noted in cases with lymph node metastasis $-57.1 \%$ which showed p53 detection in CRC correlates with a poor prognosis. However both these results failed to reach the level of statistical significance.

Among the 4 cases which showed lymphovascular invasion p53 overexpression was noted only in 1 case $(4.8 \%)$ .However it was statistically significant with $\mathrm{p}$ value of 0.03 .
We found no statistically significant correlation between p53 and age, gender, nature of specimen, tumor site, size, histological type, differentiation and grade, tumor perforation, bowel wall invasion, nodal status and perineural invasion. Similar results have been reported by Mulder ${ }^{[20]}$ and Poller. ${ }^{[26]}$

\section{Conclusion}

Our study shows that p53 overexpression is noted in advanced pT stage and in cases with lymph node metastasis which proves that p53 overexpression is a poor prognostic marker and evaluation of its expression in colorectal adenocarcinomas may prove to be useful in identifying high risk patients who may benefit from adjuvant chemotherapy in early stage of disease.

\section{Abbreviations}

CRC- Colorectal carcinoma

ASR-Age Standardised Ratio

RHC- Right Hemicolectomy

LHC - Left Hemicolectomy

$\mathrm{SC}$ - Sigmoid Colectomy

LAR- Low Anterior Resection

APR-Abdominoperineal resection

TC- Total colectomy specimens

H\&E- Hematoxylin and Eosin

IHC-Immunohistochemistry

APES- Amino Propyl Triethoxy Silane

SPSS- Statistical Package for Social Sciences

WD-Well differentiated

MD- Moderately differentiated

PD- Poorly differentiated

\section{Acknowledgements}

\section{A.Venkatesan , Dr.Deepu.R}

\section{References}

1. Ferlay J, Soerjomataram I, Ervik M, Forman D, Bray F .GLOBOCAN 2012 .Estimated Cancer Incidence,Mortality and Prevalence World wide IARC Cancer Base, Lyon:IARC Press2012.Available from http://globocan iarc.fr.

2. Arnold M, Sierra MS, Laversanne M. Global patterns and trends in colorectal cancer incidence and mortality. Gut doi:10.1136/gutjnl-2015-310912 .

3. National Cancer Registry Programme. Population based cancer registries 2004-2005. New Delhi: Indian Council of Medical Research; 2008.

4. Gandhi V, Mehta K, Grover R, Pathak S, Aggarval BB; Multi targeted Approach To Treatment of Cancer, Part III, Chapter 13 -Prevalence of Gastrointestinal Cancers in 
India,. Springer Cham Heidelberg New York London. page 217-234

5. Manne U, Heisi L. Weiss, Russel B, et al. Nuclear accumulations of p53 in Colorectal Adenocarcinoma: Prognostic Importance differs with Race and Location of tumor. CANCER 1998; 83: 2456-67.

6. Ilyas $\mathrm{M}$, Hao XP, Wilkinson $\mathrm{K}$, et al .Loss of Bcl-2 expression correlates with tumor recurrence in colorectal cancer.Gut.1998;43:383-387.

7. Mardi K, Sharma M, Bhardwaj M, Rao M. p53 expression in colorectal carcinomas and its correlation with clinicopathological parameters. Clin Cancer Investig J. 2017;6:26-9

8. de Menezes HL, Juca MJ, de A Gomes EG, Benecio L Bulhoes BP Nunes, Coata HO, Delcio Matos. Analysis of immunohistochemical expressions of $\mathrm{p} 53, \mathrm{Bcl}-2$ and $\mathrm{Ki}-67$ in colorectal adenocarcinomas and their correlations with the prognostic factors. Arq. Gastroenterol. 2010;47:2

9. Georgescu CV, Saftoiu A, Georgescu CC, Ciurea R, Ciurea T. Correlations of Proliferation Markers, p53 Expression and Histological Findings in Colorectal Carcinoma. J. Gastrointestin Liver Dis .June 2007;16(2):133-139.

10. Shanmugam C, Katkoori VR, Jhala NC, Grizzle WE, Siegal GP, and Manne U. p53 Nuclear Accumulation and Bcl-2 Expression in Contiguous Adenomatous Components of Colorectal Adenocarcinomas Predict Aggressive Tumor Behavior. J Histochem Cytochem. 2008 Mar; 56(3): 305312.

11. Prasad GR, Banda S. Evaluation of Immunoreactivity of p53 in Colorectal Adenocarcinomas. National Journal of Laboratory Medicine. 2017 Jan;6 (1): PO28-PO32.

12. Hegazy A, Daoud SA, Ibrahim WS, El-Atrebi K, SakerM and Abdel-Wahab N. Role of Ki-67, P53 and Bcl-2 in Advanced Colorectal Carcinoma (Histopathological and Immunohistochemical Study). Academic Journal of Cancer Research 2014; 7 (3): 168-172.

13. Compton CC, Fielding LP, Burgart LJ. Prognostic factors in colorectal cancer: College of American Pathologists Consensus Statement 1999. Arch Pathol Lab Med 2000; 124: 979-994.

14. Compton CC, Fenoglio-Preiser CM, Pettigrew N, Fielding LP. American Joint Committee on Cancer Prognostic Factors consensus conference: Colorectal Working Group. Cancer 2000; 88: 1739-1757.

15. Frank A. Sinicrope, San Bao Ruan, Karen R. Cleary, Clifton Stephens L, Jack Lee J , and Bernard Levin .bcl-2 and p53 oncoprotein expression during colorectal tumorigenesis. 1995;Cancer Res;55:237-241.

16. Washington MK, Berlin J, Branton P, et al .Members of the Cancer Committee, College of American Pathologists . Protocol for the Examination of Specimens From Patients With Primary Carcinoma of the Colon and Rectum. Arch Pathol Lab Med. 2009 Oct; 133(10): 1539-1551.

17. Baxter NN, Virnig DJ, Rothenberger DA, Morris AM, Jessurun J, Virnig BA : Lymph node evaluation in colorectal cancer patients: A population-based study. J Natl Cancer Inst.2005; 97: 219-225.

18. Betge J, Pollheimer MJ, Lindtner RA, et al : Intramural and extramural vascular invasion in colorectal cancer: Prognostic significance and quality of pathology reporting. Cancer 2012;118: 628-638.

19. Pages F, Berger A, Camus M, Sanchez-Cabo F, Costes A, Molidor R. Effector Memory T cells, Early metastasis, And Survival In Colorectal cancer. N. Engl.J.Med. 2005 ;353:2654-2666.

20. Mulder J.W.R, Baas I.O, Polak M.M, Goodman S.N and Offerhaus G.J.A, Evaluation of p53 protein expression as a marker for long-term prognosis in colorectal carcinoma. Brit.J.Cancer 1995;71:1257-1262.

21. Belluco C, Guillem J.G, Kemeny N, et al .p53 nuclear protein overexpression in colorectal cancer:a dominant predictor of survival in patients with advanced hepatic metastasis.J.clin. Oncol.1996;14:2696-2701.

22. Buglioni S, D'Agnano I, Cosimelli M, et al . Evaluation of multiple Bio-Pathological Factors in Colorectal Adenocarcinomas: Independent Prognostic Role of p53 and BCL-2. Int.J.Cancer (Pred Oncol).1999;84:545-552.

23. Ashar KM, Patel SC. Correlation of proliferative marker (Ki67), p53 expression and histomorphology in colorectal carcinoma. International Journal of Biomedical and Advance Research 2016; 7(12): 597-603.

24. Lanza G, Maestri I, Dubini A, et al .p53 Expression in Colorectal Cancer Relation to Tumor Type, DNA Ploidy Pattern, and Short-Term Survival. Am J Clin Pathol 1996;105:604-612.

25. Ofner D, Maier H, Riedmann B, etal. Immunohistochemically detectable p53 and mdm-2 oncoprotein expression in colorectal carcinoma: prognostic significance. J Clin Pathol: Mol Pathol 1995;48:M12-M16.

26. Poller DN, Baxter KJ, Shepherd NA. p53 and Rb1 protein expression: are they prognostically useful in colorectal cancer? Br J Cancer 1997;75:87-93.

*Corresponding author:

Dr. Varshaa Chithrra, Final year PG in MD Pathology, Dept.of Pathology, SRM Medical College Hospital and Research Centre, Kattankulathur, Chennai-603203, Tamil Nadu, India

Phone: +91 9846407412, 8129097358

Email: drvarshadeepu@gmail.com

Financial or other Competing Interests: None. 